$16 \mathrm{May}, 1882$.

Sir F. J. BRAMWELL, F.R.S., Vice-President, in the Chair.

(Paper No. 1879.)

\title{
"On the Various Systems of Grinding Wheat, and on the Machines used in Corn-Mills."
}

By William Proctor Baker.

Ir is impossible within the limits of a short Paper to discuss exhaustively the processes, and fully to describe and criticise in principle or detail the machines used in modern corn-mills; such a discussion could be compassed only in a volume of considerable dimensions. All that will be attempted in this Paper is a statement of the conditions under which the miller's business has to be carried on in the United Kingdom, as compared with those countries from which flour is largely imported, followed by a general sketch of the prominent features of the processes in use, and of the machines employed. Except in one or two cases, mention or description of particular machines will be avoided, and the aim will be to enumerate the elements which are common to the design, or the objects which are sought to be attained by classes of machines. There would have been space perhaps to describe, with the aid of drawings, one typical machine in each class ; but when in almost every case there are machines of merit competing in the market, it appeared that in selecting one, injustice may be done to the inventors and makers of those not named. There is no machine in use of which detailed accounts have not been published in the milling or engineering periodicals, which are easily accessible ; so that, as any drawings to accompany this Paper would not be in illustration of novelties, they have been omitted.

Although there is abundance of materials whence to construct a history of the art of corn-milling from early periods, yet it is not

1 The discussion upon this Paper was taken together with that upon the two following ones. 
expedient to attempt to give a description of all the various systems, nor of all the machinery of which records exist. A rapid glance may however be taken of the modes used by the ancients to obtain flour from wheat. The earliest known implements in use were the pestle and mortar, in which the grain was pounded by hand, and rollers, between which and a hard stone tablet the grain was crushed by hand-pressure. The Eastern hand-mill, still to be seen, is the first recorded instance of the grinding operation by millstones on the same principle as is now practised. At the commencement of the Christian era these had been developed into the form to be seen among the remains at Pompeii ; the lower millstone was fixed, and, among the Romans, the grinding surfaces were sometimes conical, sometimes spherical, sometimes flat; the upper, or moving stone, was turned in one direction by a lever attached to its periphery, first by hand, then by animals, afterwards by water-power; the notable improvement over the pestle and mortar, or rolling process, being that the corn to be ground was distributed between the grinding surfaces through a hole made for the purpose at the centre of the upper and revolving stone; and as the meal, i.e., the ground corn, escaped from between the grinding surfaces at the edges of the two stones, a continuous stream of corn could be passed between the stones, and the operation could be carried on so long as the upper stone was caused to revolve. After the grain had been thus crushed or ground, the, fine particles of flour were separated from the imperfectly ground fragments by hand-sieves, formed of pierced skins or parchment. The partly-ground fragments of wheat were again and again passed between the millstones, until as much flour as practicable had been extracted. The residuum was the skin of the wheat, called "bran." These millstones and sieves are the germs from which have been gradually developed the elaborate machinery of the modern cornmill. Coming down to a thousand years ago, the millstone then in use was probably of the same type as the millstone of the present day. The features which are identical between the two periods are these: the lower, or bed-stone, fixed; the upper stone, or runner, made to revolve by power; the grinding surfaces horizontal and nearly parallel to each other; the runner fixed to, or hung loosely on the top of a vertical spindle passing through the centre of the bedstone; the foot of the spindle carried on a bearing so contrived that it may be raised or lowered at the wish of the operator, while the spindle is in motion for the purpose of increasing or diminishing the distance between the grinding surfaces of the two millstones; means of regulating the volume of the stream, or 
" feed," of corn, and its even distribution between the grinding surfaces; the establishment of a sufficient speed of rotation to throw out the ground material; the enclosure of the millstones in a tub or case; and the delivery of the meal from it by a pipe. The method of sifting the meal after it was ground by hand-sieves seems to have been the only one used down to recent times, but the material for covering the sieves varied among different nations; thus the Egyptians used rushes and papyrus; while it is recorded that pieced skins and parchment, horsehair and linen, were employed in the first century of the Christian era. Among the Romans the grinding, sifting, and baking operations were all conducted in one house. In London, however, in the fourteenth century, it is clear from the regulations to be found in the "Liber Albus," that the miller had a separate establishment from the baker; that the miller ground the corn to meal; that the meal was delivered to the baker, who sifted it by sieves or bolts at the bakery, and the master-baker was bound to instruct his men twice a year in the mode of bolting. In Shakespeare's time the word "bolting," as applied to the sifting of meal, was familiar, but whether there was any machine used for the purpose besides the hand-sieve does not appear. Flour-dressing machines, as those machines are called which perform sifting operations, may therefore be considered the product of comparatively modern times, though it is curious to observe that Americans name all such machines "bolts."

Attention has been lately called in many ways, and especially by the Exhibition of Mill Machinery last year, to the revolution that has been taking place in the art of milling, and it has been erroneously supposed that, first the Hungarians, and afterwards the Americans, had, by some imaginary secret processes, been able to eclipse the corn-millers of all other nations. It may be well to state here that there are no such secret processes, but that the Hungarians have produced flours still unsurpassed in excellence by skilful manipulation of their native wheats (which, though yielding very bad flour when ground by old methods, possess admirable qualities), and that the Americans, by recently adopting similar, but not identical processes, modified to suit their native wheats, have been able to make flours second only to the Hungarian in quality; and it should be understood that out of similar materials, millers in England could, by using similar machinery, attain the same results. As however the Hungarian and $\Lambda$ merican mills buy up all the special qualities of wheat from which the finest flours are made, these materials are not obtainable by the English flour 
makers: and it should also be understood that the conditions of the trade are very different in England to those of either of these other countries; for in England the millers work chiefly for the supply of the district near their mills, and each mill must be adapted to produce the particular sort of flour consumed in that district; and in some districts the taste is entirely for fine white bread, in others for medium, and in others for low brown bread. As a rule the demand in each district runs on only one class of flour, and the local miller is obliged to manufacture that sort to meet the local demand.

The processes used in Hungary are based on the principle of dividing the flour produced from the same wheat into eight or ten or twelve different qualities. The fine qualities, which command very high prices, find their market in various central and western parts of Europe, and in certain parts of the United Kingdom, and in Brazil, while the medium and lower qualities find a local consumption as well as a demand throughout the south-east parts of Europe.

In America, the recently-adopted practice is to divide the flour made from one sort of wheat into several grades of quality, fewer however than in the Hungarian method, for which in like manner a sale has to be found in various and perhaps widelyseparated markets.

There is never any difficulty in selling flour of the medium grades, which is the flour almost universally in demand; but for flour of the finest quality and highest price the demand is restricted, and flour of the lower grades is at times only to be disposed of at comparatively very low prices, although all the markets of the world may be open to the miller who knows the quality in demand in every district and can send appropriate flour to each. Now the English miller must manufacture for local demand ; for, having already incurred the cost of freight and carriage on the wheat in bringing it to his mill (wheat which he may have to buy in competition with his foreign competitor), he cannot afford to pay another freight on the flour to carry it to a distant place of consumption where he will meet again the competition of the Hungarian or American miller, who can send flour direct to the same place and thus incur only one freight on it (and that freight less than the wheat freight). Thus it is that every miller in England must work for local demand, and must seek to adopt a system that will produce the quality of flour required by the taste of his own district, and he cannot, like the American or Hungarian, depend on finding an outlet 
for his manufacture in distant markets. This is the reason that American and Hungarian systems have not been extensively adopted in this country. Great caution is required not to be deluded by the temptation to manufacture a superior quality of flour without the assurance that it can be sold (if it can be sold at all) at a superior price; and in forgetfulness that the making of the superior flour entails also the production of a quantity of inferior flour that will be a drug in the local or any market within reach. And the millowner in England has another difficulty to contend with. It may be roughly calculated that twothirds of the wheat henceforth to be ground in England will be of foreign growth. Foreign wheats differ materially from each other and from home-grown wheats in character, and in the quality of flour produced from them; but the miller is obliged, as has been stated, to work to the standards of quality of flour of his district whatever they may be; that is to say, that he must produce, whatever may be his materials, a certain fixed quality of flour. The supplies of English wheat differ in quality in each district from year to year in sympathy with the variations of the weather: foreign wheats from the same district change less than do the English from year to year; but the foreign wheat available to the miller not only varies in quality from year to year, but the supply also varies in different parts of the same year, according as each country sends forward its produce; thus it may be that at one time American spring wheat may predominate; at another, American winter wheat; at another, Californian, Oregon or Chili wheats may fill our stores; at another, Russian; and so on, Indian, Australian, German, French, each in its turn, and each differing from all the rest in character. As all these wheats require different treatment in grinding to produce the same character and quality of flour, the English miller, therefore, having to produce constant qualities of flour, requires to be equipped with such apparatus as will enable him to deal equally well with all these differing materials, for to conduct his business profitably he must be able to work up whatever may happen to be in proportion to its quality the cheapest wheat of the day. $\mathrm{He}$ must adopt a system applicable to all sorts of wheat, and not one that is profitable only when employed on only one description, such as some of the foreign systems are. On the other hand, his foreign competitor has no such difficulty. He draws his supplies from the districts immediately surrounding his mill; they vary only from year to year according to the harvest, and there is no wide difference in the character of the grain between one year 
and another, and the quality is the same from day to day throughout the year; so that his task is only to select the best method of dealing with one particular sort of wheat, and of that wheat to obtain the produce in the form that will fetch most money without regard to the wants of the population at his door, for he has all the accessible markets of the world open to him. The most profitable system of flour-making is that which causes wheat to yield the largest proportion of high quality fine flour, provided always that a sufficiently high price can be realised for it. But it is not difficult to overdo the market with fine flours, that is, to produce more than the demand will take off at sufficiently high prices; and this is what has happened: first to the Hungarians, who are unable now to keep their mills profitably employed because their flour was displaced to a great extent by the Americans, whose materials were cheaper; and then to the Americans, for, attracted by the profitable nature of the business, they have erected mills on a very large scale depending on finding an outlet for any quantity of the finest flour, and it soon happened that the shipping ports were glutted with these fine sorts for which adequate prices could not be obtained, while there was not enough of medium qualities to meet the demand. With these warnings it is additionally important that English millers should be cautious only to adopt such systems as will enable them to make profitably those particular qualities of flour for which a demand exists in the district supplied by each mill, and that engineers should, in designing mills, carefully adapt the machinery to be supplied for that purpose. A mill suitable for Glasgow, where fine flours are largely consumed, would not be suitable for London, where the bulk of the demand is for medium grades. Each district has its own peculiarities, and one universal type of mill will not succeed everywhere, either with regard to the qualities of flour it is calculated to produce, or with regard to the raw material that may be available. The selection of a system and of the machinery must be matter for the judgment of the millowner, who alone can know the requirements of his trade as to qualities of flour, and what are likely to be the sorts of wheat which he will have to operate upon; and he should turn a deaf ear to the blandishments of the mill-furnishers who promise that marvellous profits shall arise if only the special system they have an interest in advocating be adopted, whatever may be the commercial situation of the mill.

It will be useful to define more particularly what has to be accomplished in wheat-grinding, and then to discuss generally the 
various systems in practice. The shape of the wheat-corn is familiar, and it is well known that, physically, it consists of the germ, the flour-yielding body of the corn, and the skin or hull which encloses it. The flour-yielding body is built up of minute cells of irregular and many-sided shapes, containing the starch and gluten of which flour consists. The hull or skin is closely attached to the cells next it, and it cannot be stripped off like the skin of a nut or the hull of an oat. The problem for the miller is how, in the simplest, cheapest, and most complete manner, to separate the germ and skins of the wheat-corn from the flour; and, further, as the quality of the flour varies according to its position in the wheat-corn, how to separate the richest and best of the flour-containing cells from those that are inferior. In consequence of the adhesion of the skin of the wheat to the interior it is not possible in practice to make an absolutely perfect separation of the parts; so that his aim must be to obtain from a given weight of wheat the greatest quantity of the best quality flour the wheat will practically yield, and the least quantity of "offal," as the skins and germ are technically named.

It should be observed that the value of offal in the market is very little enhanced by leaving flour in it; while the value of flour is much diminished by the slightest admixture of offal or "bran." The financial importance of the complete separation of the flour from the offal is obvious from the consideration that, while flour varies (at present prices) from $10 \mathrm{~s}$. to $23 \mathrm{~s}$. per cwt., offals are worth only from 48. to 6s. per cwt. The most important point is therefore not to permit any particles of bran to be mixed with the flour, because such a mixture seriously reduces the value of the flour; and, that point secured, then to be careful that no loss is made by leaving valuable flour among the offal to be sold at the very low comparative price which it fetches.

Up to within about ten years ago the general system in this country and in America was what is termed low grinding. It is assumed that the form and arrangement of millstones is well known, and low grinding means that the grinding surfaces of the two stones are brought so closely together as to crush at one operation all the interior of the grain into the powder known as flour, while the exterior skin is spread out into flat flakes (called bran), off which all the floury particles have to be scraped. The skilful operator under this system so managed the arrangement of the stones that while he achieved the object of cleaning the bran, yet he avoided as much as possible the breakage of the skin for two reasons-(1) because the less broken it is, the less are the particles 
likely to pass with the flour through the sifting material; and (2) because in commerce the larger the flakes, the more valuable is the bran. It is indeed possible by very well-arranged millstones to perform the grinding so completely that the millex has no more to do than to divide the flour from the offal by sifting, or dressing the meal, as the millers' term is. This is the most simple form of grinding, and, even with all the latest improvements, is that which is best adapted to the requirements of those localities where the wheat used has a somewhat tough skin, and where at the same time the demand runs on a medium or somewhat low class of flour. Wheat, however, varies very much in the toughness of the skin, and while the skin of some wheats is very difficult to break, in the case of other sorts the skin is as brittle and friable as the interior of the corn. Conspicuously this is the characteristic of the wheats produced in Hungary and in the North-Western States of America; and rich as these wheats are in all the elements which go to make good flour, their skin is so brittle that any attempt to grind the interior parts into flour by low grinding has the inevitable result of breaking up the skin at the same time, and thus producing a flour in which there is a large mixture of minute particles of bran, and therefore flour of very low quality. These particles of bran have the effect not only of colouring the bread by the admixture of so many specks, but produce also a certain secondary fermentation, which destroys some of the constituents of the flour and discolours the whole of the bread. The Hungarians, finding it impossible to keep the skin unbroken, and the kernel of their wheats when exposed to the action of the millstone, on account of their structure and brittleness, having a tendency to break into small fragments, rather than into flour, like soft wheats, availed themselves of this circumstance, and instead of seeking to make flour at one grinding operation, they regulated their millstones so as just to crack or break up the wheat into small gritty particles, named by the Germans "gries," by the French "gruaux," and in the United Kingdom "semolina." The breaking operation had to be repeated several times, and the semolina obtained by the successive breaks, mixed as it was with small particles of bran, was exposed to winnowing operations, which got rid of the bran and left the small cellular fragments of the interior of the wheat in a pure state, free from bran. The pure semolina could then be crushed down by millstones into fine flour. Similar principles have been applied to the treatment of the hard wheats of the North-Western States of America, with some modification of the process. Between the low grinding, which is the most simple, and 
the Hungarian system, which is the most elaborate in detail, various systems have been developed, and are known as high grinding, halfhigh grinding, new process, and gradual reduction, and modern machinery has been contrived to achieve some special object in connection with one or other of these processes.

Whatever may be the subsequent treatment of the meal, the mode of grinding the wheat is what gives name and character to the process, and it is necessary now to give a brief sketch of the leading systems.

In low grinding the surfaces of the millstones are kept sharp by being dressed by a mill chisel. The working surface of a millstone after running a few days becomes smooth and polished, and it will then no longer scrape the floury particles off the bran; and the dress takes place by making cracks or cuts with a mill chisel, varying in width and depth according to the quality of the millstone and the style of grinding; these cracks are made parallel to each other, and vary in number from twelve to twenty-five per inch. The man who performs the work is called a stonesman, and he is paid at a higher rate than workmen in any other department of the mill, as the success or failure of the grinding operation depends upon his skill and judgment in dressing the stone. The furrows are the channels made in the stone, and the edges and surfaces of these have to be kept sharp and true in the same way as the plane surfaces of the stone. In grinding, the surfaces of the stones wear away unequally, and one of the chief duties of the stonesman is to keep the surfaces perfectly true, by cutting off any hard and high spots, and by dressing very lightly or scarcely touching any hollow places. As the surfaces of the stones approach each other in this system very nearly, when running, it is obviously of the greatest importance that they should be true. The object is to clean the bran at one operation, and to break it as little as possible, for the less it is broken, the less likely are there to be minute particles of it which would pass with the flour through the meshes of the sifting apparatus, and thus become inextricably mixed up with the flour. By this system, although it is not possible to avoid making any gritty particles (called middlings), yet nearly the whole of the interior of the corn is reduced to fine flour at one grinding, and nothing remains but to dress (that is, to sift) the meal and divide it into flour and the different sorts of offal, when the operation is complete. It is the most simple system, and the least expensive in installation and working, and requires the least space. And it is more than doubtful whether the results obtained by it from wheats, such as our native English wheat, in a 
district where flour of moderate quality is desired, can be surpassed by the more elaborate procedure of systems of more recent invention, although it is the fashion to sneer at those who practise it as "non-progressive."

In high grinding the object of the operator is just the reverse of the low grinder, viz., to avoid as much as possible making flour when the wheat passes through the stones for the first time. The idea is that the corn should be rolled abroad under very little pressure from the millstone, so that while the bran is detached from the floury particles of the interior of the grain by gentle rubbing, those floury particles should be left as much as possible in the gritty condition of semolina or middlings, while the bran is to be left to the greatest extent possible unbroken, and the miller is not careful that it should be absolutely clean. It is therefore needful in this system either to regrind the bran for the purpose of cleaning it, or to leave some flour adhering to it. If the bran be re-ground, the resulting flour is always of very low quality. If flour be left adhering to the bran there is waste of flour. The desire is to make as little flour as possible, because all the flour produced at the first grinding is more or less mixed with specks of the branny coat; on the other hand, by the winnowing operation, to which the middlings are afterwards subjected, it is possible in theory, though in practice difficult, to get them purified from all these specks, and the middlings can then be broken down into flour by a subsequent grinding, and that flour is nearly pure, and consequently of high relative value. Instead, therefore, of keeping the surface of the millstones sharp, the high-grinding miller seeks to keep them extremely true, and then rubs them down to an even surface not actually polished, but very slightly rough. In treating the meal after this grinding, the offals are divided from the flour and the middlings, the middlings are divided from the flour; they are then purified by winnowing, and being reground and the resulting meal sifted, a very fine flour is obtained; the bran also being reground produces a very poor flour. The produce, therefore, is in the form of flour of at least three qualities, viz., the flour produced at the first grinding, the ground middlings, and the bran flours, instead of one quality as in the low grinding system; and thus high grinding can be profitably practised only when a market at a comparatively high price can be found for the fine flour. All depends on this, for it must be observed that the flour produced at the original grinding is not so good as low grinding flour from the same wheat, because it does not contain the very good flour 
made from the middlings, and that the bran flour is a very inferior article. So that the profit of this system of working depends entirely on the maker being able to dispose of his fine flour at a high price, and to find a market at not too low a price for the inferior and bran flours.

Under another system known as half-high grinding, while the bran is cleaned at one operation, middlings are produced to a larger extent than in low grinding, and being purified and reground, are mixed with the original flour with the effect of improving its quality, or the middlings may be sold separately at a higher price. This appears to be the plan best adopted for the greater number of mills.

Gradual reduction, however, is the system under which undoubtedly the highest quality of flour can be manufactured. The object of the operation is to break up the whole of the interior of the corn into semolina, to purify and repurify the semolina, to classify it according to its quality (for it varies in quality according to its position in the corn), and to crush it into flour of various grades. By these operations the finest known flours are made, and the profits depend on wheats of fit character being obtainable, and on sales of the finest flours at very high prices being practicable. But the demand for such flours is limited, as the Hungarians have found to their cost by the pressure of the American competition, and as the Americans discovered by having had their markets glutted with quantities of fine flour far exceeding that for which there was a demand, and by the consequent depression of the prices of such qualities below remunerative rates. It has been ascertained that the system does not pay, if all the separate qualities be mixed together to form a medium flour, for its profits depend on the relatively high prices paid for the highest quality flours: slight improvements in quality in high grades being marked by much larger increments of price than similar differences in medium sorts. Moreover, the system is not adapted for soft tender wheats, but only for such wheats as are hard and flinty in texture, brittle enough to break into fragments instead of being crushed by pressure into powder or flour. The operations are now carried out chiefly by rollermills, and the following is the process. The wheat is led between a pair of rollers, which are fluted or grooved, running at a differential speed, and set at a distance apart slightly less than the diameter of the wheat corn; the corn is thus slightly cracked; the cracked corn is sifted to remove any dust that may have been disengaged; the cracked corn is then led to another 
set of rollers working somewhat closer together, which carry the breaking of the grain farther than the first pair; the resultant meal is again sifted, and this time fragments of the interior of the corn are obtained in the shape of gritty particles, which are accompanied by some flour; and these alternate cracking and sifting operations are again and again repeated until all the interior portion of the grain has been disengaged from the bran. The roller-mills are so arranged as to increase gradually the pressure on the corn at each successive cracking, and the grooves of the rollers are finer and sharper so as gradually to scrape all the flour-bearing particles off the bran. The great object is to avoid the production of any flour during these successive breaks, and to obtain as much as may be of the interior of the wheat in the form of gritty fragments, known as semolina or middlings. It is impossible to avoid, even with the wheuts best adapted for the process, the production of a certain quantity of flour in these successive breaks, and after the separation of the bran, which generally has to be finally cleaned by millstones, a meal remains consisting of semolina of various sizes, particles of bran of corresponding sizes, and flour. As for the purpose of purifying the semolina it has to be passed through winnowing machines, and as the flour would be blown away if taken into those machines, the first step is to separate all the flour from the semolina and particles of bran by sifting. Next, as the winnowing is a very delicate and nicely adjusted operation, it is necessary to divide the semolina into gradations of size, as the amount of wind which would be fit for the larger sizes would blow away the smaller. This operation is effected by means of silk or wire cloths adapted for the purpose. The semolinas are then passed through purifiers, which remove all the branny particles that are lighter than the semolina, though they may be of the same size, and are so nicely adjustable that they are capable of separating the more dense semolina from that which is less hard and compact, and also semolina which has particles of bran attached to it from pure semolina. In this way the semolina can be purified and sorted into various qualities, and it then remains to crush them down into flour. This is accomplished by millstones, or by rollers of smooth surface working under considerable pressure. The flour obtained is sifted out of the meal, and the residue is again treated as was the original semolina, and the operation is repeated as often as may be required to obtain the whole of the flour. The flour, however, from each successive grinding becomes worse. And thus it is that from the original breaks of the wheat, from each quality of semolina, and from the 
successive grindings of semolina, various qualities of flour are obtained. As, however, has been already stated, this system can be used with financial success only with appropriate wheats, and when a market can be obtained at remunerative prices, which must be comparatively high for the fine flours. For as the process is a costly one in consequence (1) of the larger size of the building required for the various and complex machinery employed; (2) of the cost of the extra machinery; (3) the cost of extra labour and skilled and expensive superintendence required to conduct the elaborate details of the operations, it cannot compete in point of the cost of manufacture of the ordinary grades of flour with either the low grinding or half-high grinding systems, as those are much more economical processes to conduct.

The delicacy and the exactitude, and even the intricacy, of all the separate processes of the gradual reduction system, as compared with the simple proceedings in the low grinding system, no doubt give the former a greater charm for the scientific and mechanical mind, but the cheapness and ready adaptabilty of the latter to working nearly all descriptions of wheat will give it a vitality not perhaps expected by the advocates of progress, and it may safely be predicted that no time can be assigned when the sound of the millstone shall be heard no more.

A sketch of the various systems of grinding having been given with a careful avoidance of technical details, it remains to attempt a general survey of the great variety of machines now used in carrying out the processes that have been briefly described. Many machines, constructed on the same principles and intended to accomplish the same duties, differ only slightly in matters of detail, but the difference, although apparently minute, is often sufficient to distinguish a successful from an unsuccessful machine.

It is proposed to describe the general features only of classes of machines, as it is impossible to deal with the merits and defects of individual machines in the space available. The machinery in use may be classified according to the work to which it is applied, and the classes would, corresponding to the rotation of the processes, be thus divided:

1. Wheat cleaning and separating.

2. Grinding.

3. Sifting or dressing.

4. Semolina or middlings purifying. 


\section{Wheat cleaning aNd separating.}

The wheat of commerce as delivered to the corn miller is not in a fit state for grinding, because even when the grower is provided with threshing and winnowing machinery of the best agricultural type, and uses it in the most careful manner, he is unable to deliver the grain free from admixture of dust, chaff, or seeds; and there is a gradation in the amount of matters other than grain, found amongst the wheat from that proportion which is contained in the carefully cleaned corn, down to that which exists in corn brought from foreign countries where the peasantry are unskilled in the use of machinery. Besides the foreign matters delivered with the wheat by the farmer, there is also to be found in imported wheats pieces of wood, sticks, rope, twine, matting, nails, and a variety of articles, which are introduced during the conveyance of the wheat, either in carts, barges, ships, or railways. In wheat from some countries stones are always to be found mixed with the grain. It is therefore evident that very complete arrangements for getting rid of all these impurities is the first necessity in every mill.

It is desirable in well-managed processes of cleaning to begin by getting rid of all easily separable matters, such as dust, and other matters that are larger than the wheat corns, and those that are smaller. Such things are readily removed by oscillating or rotating sieves, covered with wire gauze or punched metal plates, with meshes or holes that at one part of the machine let the wheat through and throw out the larger articles, and at another part let all the small things through and retain the wheat. There still remain, however, chaff, shrivelled grains, husks of grains, the interior of which has been eaten by weevils, and bits of straw and chaff. In order to remove these the corn is caused to fall in a stream through a current of wind, the intensity of which can be regulated by valves, and all these light particles are blown, or by preference sucked, away. There remain, however, mixed with the wheat other matters, such as stones, particles of iron, and seeds of the same weight as the wheat, but round instead of oblong, and oats, which are much longer than the wheat. For the removal of each of these where present a distinct machine is required, and efficient machines leaving nothing to be desired exist for each purpose. For the removal of iron, the quantity of which mixed with wheat is large, and which has been increased by the adoption of wire- 
binding reaping machines, the wheat has to be passed in a stream over an arrangement of powerful magnets. There are some descriptions of wheat, Egyptian for instance, which contain so much mud, and the grains are so coated with dirt, that nothing short of washing thoroughly will cleanse them. Powerful machines of various types are made which accomplish this operation. The grain becomes soft in the washing process and washing necessitates drying, and the drying is partly done by centrifugal machines, but the application of heat is needed before the corn can be made sufficiently hard and dry for grinding. Kilns, or steam, or hot-air drying machines are therefore needed at this stage. The wheat, having been so far freed from extraneous matters as the machines already mentioned will permit, still has among it particles of hard mud, and there is loose dust, and dirt and dust attached to the coat or lodged in the crevices of the grains. To detach and remove this dirt, and to remove loose particles of skin and, so far as possible, the hairy end of the corn, and to break up any bits of mud, strong friction is applied, and the most approved practice is to use, not, as formerly was the case, machines with rough surfaces, but rather such as depend for their operation in rubbing the corns one against another. These machines are provided with air currents which carry away from among the mass of grain the dust as fast as it is detached. But after this process there still remains dust attached to, or lodged in, the crevices of the corns, and finally to polish the grain it is subjected to machines which, while turning it over in all directions, brush it under a slight pressure. The grain thus treated is at last ready for grinding.

Here it may be well to remark that decortication by a variety of methods has been attempted, but that on account of the irregularity of the shape of the wheat corns, and of the existence of the crease or fold from which the skin can by no means be removed, complete decortication has never been accomplished, that it is an operation which requires a large expenditure of power, and that in any case it must be accompanied with considerable waste in consequence of the breakage of the grains. So that it does not appear probable, or indeed possible, that the separation of the skin of the wheat from its kernel by decortication will ever be accomplished by mechanical means. When the skin is spoken of it must be understood that the entire skin, consisting of five layers, is intended; of these the outer skins are easily detachable, but as they are composed of woody fibre only, and have no chemical action in the bread-making process, 
their removal alone is not attended with any advantage worth the trouble and cost.

The only further operation to which wheat is subjected in some cases, where a preliminary crushing, or rather cracking, by rollers is adopted, is to size it; that is to say, to divide the small corns from the large, so that each may be separately treated.

\section{Grinding.}

The wheat, having thus been thoroughly cleaned, is ready for grinding, and the grinding is accomplished either by millstones, by rollers, or by disintegrators, or by a combination in successive stages of these means of reduction. Millstones are so well known, and every detail of their arrangement, so far as mechanism is concerned, has been so frequently discussed, that it is not needful to enter here upon that part of the subject. But there has been so distinct a change in the last few years with regard to the dress (which is the term used for the mode of furrowing or grooving the millstone), that it cannot be passed over in silence. In the low-grinding process little change has been made; but in all the high-grinding systems, however modified, wherever the desire has been to increase the produce of semolina, or middlings, in proportion to the produce of flour, there has been a complete reversal of the old principles of dress; for in the old plan, however the shape of the furrows may have differed, the area of the furrows was not more than one-fourth or one-third of the plane surface, or lands, of the stone; in the new practice, while the different plans are legion in number, they all agree in increasing the number and area of the furrows, leaving a very small proportionate area of lands. The number of edges. of furrows thus being multiplied, and the plane areas, which are the grinding or rubbing surfaces, reduced, and the surfaces set farther apart when the stones are at work, the tendency of the action of the stones is clearly to break the grain into small fragments, rather than to crush and rub it into flour. And that occurs also which may have been expected, viz., that the bran is more broken up and is not scraped clean, as in the low-grinding system.

Roller-mills are, however, the novelty of the day, and their use has been much extended in the last two or three. years. The designs and construction of these machines are most various, and each type is probably conspicuous for some advantage, as it is also for some drawback or defect. As it is impossible to describe even 
a tithe of the different varieties, and to discuss the details of them, it appears to be more useful to consider generally what are the uses of roller-mills, and,what are the essential and important features in their construction.

A roller-mill, then, is destined for the breaking down or reduction of either wheat, or semolina, or middlings. But the machine that is adapted for one purpose may be by no means the best to accomplish another, and even to-day the subject has scarcely more than emerged from the experimental stage, and there has not been time since their general employment to test completely and conclusively the advantages and disadvantages attending the use of all the various forms of machines. And an expression of regret cannot be omitted that these roller-mills should be as they are, with very few exceptions, the production of foreign workshops. A very large proportion of the roller-mills at work in this country have been made in Switzerland and Hungary, and a few come from America. The introduction of the form of roller which has recently found so general an application is due to a Swiss, Mr. Wegmann; and it was in the mills at Budapest that the employment of them on an extensive scale was first made.

What is required of a roller-mill in breaking wheat is, that it should leave the bran, or skin, as far as possible intact, and that it should reduce the kernel, not immediately to flour, but to the small fragments known as semolina, and should avoid crushing any of the semolina to flour. The function of a roller-mill in treating semolina is either to crush it at once to flour or to reduce it to smaller sizes to such an extent as the operator may desire.

The first point to consider is the material from which the rollers themselves should be made. The material should be homogeneous, so as to secure the permanence of an even surface, and to avoid its wearing unevenly, either in large spaces or in small pits or grooves, or so that unworn prominences should be left; it should be sufficiently hard not to receive damage from pieces of quartz or metal (such as nails) passing between the rolls; and tough, so that the edges of the grooves cut in the surface of the rollers may not break away. It must be borne in mind that the coat of the wheat contains so much silica that the hardest materials, when in constant moving-contact with wheat, wear away. The materials employed have been cast iron, cast steel, forged steel, chilled cast iron, and porcelain; and of these, chilled cast iron and porcelain are the two which answer best. Of these two sorts the iron rollers are fitted for and used for every description of work, both as 
smooth rollers and with grooves, while the porcelain rollers do not stand the wear of breaking down wheat, nor do the edges of grooves in them stand wear; but, as smooth rollers for breaking down the softer kinds of semolina and middlings, they are by many preferred to iron on account of the porosity of the surface. Not but that it is contended (and experience confirms it) that the polished hard iron rollers, after a certain amount of use, do attain also a certain and sufficient roughness of surface. The porcelain rollers have an advantage in not being liable to the rust which would affect the surface of the iron rollers if they were for some time out of use and were unprotected, but against this is to be put the liability to fracture, from which iron rollers are free.

The dimensions of rollers, especially in roller-mills when pressure is applied, seem to be fixed within narrow limits. In order to accomplish a large amount of duty the inclination would be to construct the rollers as large as possible; but experience has shown, having regard to the size of the grain or semolina to be ground, and the desirable period of contact with the rollers, that the rollers should be of a diameter of between 9 inches and 12 inches. The angle formed by the approach of the surfaces of the two rollers is of importance with smooth rollers, for if it be too great the material may not be drawn between the grinding surfaces. As to the length of the rollers other considerations limit it. The surfaces must be absolutely true, and the longer the roller the greater is the difficulty of getting perfectly true isurfaces, and also the greater the difficulty of obtaining homogeneity. The manufacturer is thus unwilling to incur the risk of failure in attempting to make very long rollers. And in rollermills, where the work requires much crushing, there is always great difficulty in contriving an adjustment that will keep very long rollers quite parallel to each other when heavy pressure is brought to bear through them on the material to be ground; and as the pressure on the bearings is in proportion to the length of the working surfaces of the rollers, it is not surprising that in practice it has been found impossible to run the larger rollers, exerting the highest crushing power required on the material to be ground, without causing the journals of the rollers to heat to a great extent, and, this consideration apart, that the mill thus constructed absorbed an inconvenient amount of power to drive it. The length of rollers in the best machines working with pressure is therefore limited to about 15 inches, but rollers so long as 30 inches are in use in the case of grooved rollers working without pressure. 
The surface of the rollers is either smooth or grooved. If smooth the highest finish of the surface is demanded. If grooved, the object of the groove being to obtain a cutting edge, it is important that the form of the groove (as well as the material of the roller) should be adapted to keep up a sharp edge; the edges are intended to cut or break the interior of the grain into small fragments, and to scrape the bran clean. If the edges of the grooves be sharp this object is attained; if they be blunt, the cutting action becomes a bruising one, and the effect is that flour is made instead of the semolina which is desired. The number of grooves varies from eighteen to thirty-five per inch. Some rollers have been constructed with rounded grooves or corrugations, the object being to avoid cutting up the bran, and these may succeed with tender wheats, and when it is proposed to obtain flour and not semolina. The grooving varies considerably as to the widthe of the grooves, and as to their depths according to the work to be done; some grooving is parallel with the axis of the roller, some is spiral. The spiral grooving produces a scissors-like action. The spindles of the rollers should be of large diameter and the journals long; it may here be mentioned that the frames, bearings and carriages should be of very solid construction, amply provided with means of lubrication, and well protected against the entrance of dust, and that all parts should be readily accessible.

The most simple form of roller-mill is that in which a pair of rollers work horizontally side by side; to these is frequently added a second pair. Another form of frame contains three rollers. operating as two pairs, the rollers being one above the other, and the middle roller being common to each pair. Rollers work either with or without pressure. Grooved rollers may do. their work with or without pressure; smooth rollers can do their work only with pressure. Grooved rollers working with pressure must be arranged so that they cannot approach each other so nearly as to touch, for if in contact the edges of the grooving would be destroyed.

The pressure should be of an elastic kind so as to allow the roller to recede in case of an ungrindable object falling by accident between the rollers. The power of regulation of the pressure must permit delicate adjustment of increase or decrease from timeto time, and admit of its instant removal or application up ta a known fixed point. It is most important that the amount of pressure should be constant. It must also be exactly alike at each end of and at all points of the working surface of the rollers. Various contrivances have been adopted to apply the pressure, and. 
in all cases the object is gained by fitting one or both the rollers working together with movable bearings. These are operated upon either alone or in combination by wedges, screws, springs, and levers. It is impossible in a short Paper to describe in detail the various plans, or to compare their merits or demerits. The weak points are speedily found out after a short experience in actual work. Especial notice ought, however, to be directed to the ingenious and excellent contrivance of the anti-friction ring pressure arrangement invented by Mr. Mechwart, which affords the power of applying heavier pressure than any other roller-mill.

It remains to speak of the speed at which the rollers are driven; and this again has been fixed by experiment, and in the best examples appears to be limited to a periphery speed of 5,000 to 5,500 inches per minute, which for a roller of 9 inches diameter requires about two hundred revolutions per minute. In some cases only one roller of a pair is driven, the second roller acting as a cushion against which the corn is pressed, and turning by the friction; this is the case only with smooth-surface rollers, and it is also only smooth-surface rollers that are driven at equal velocities. The effect of even-speeded rollers being only to squeeze what passes between them, it is found that the resultant meal is delivered in flattened cakes; these cakes in all cases require to be rubbed abroad so as to obtain a meal that can be sifted, and for this purpose appliances called "detacheurs" are used either in eonnection with the roller-mills or as separate machines, which simply break up the small cakes. For breaking up the wheat grains, and for grinding the semolinas, differently speeded rollers are almost universally adopted; and for the reason that as the material to be ground is in contact on the one side with a slow moving surface, and on the other with a fast moving surface, a tearing action is produced which enables the grinding operation to be accomplished with far less pressure than when the squeezing action is alone relied on. The difference in the speed of the rollers varies according to the material to be dealt with. The proportions of 4 to 5,3 to 2 , and of $2,3,4,5$ to 1 are common differences; while one roller-mill has been constructed in which the fast coller runs at about five hundred per minute, and the second coller is arranged to run at two and a half revolutions per minute, but so that the contiguous surfaces of the two rollers revolve in opposite directions.

It is most important that the details of the feed to the rollers should be arranged so as to allow of delicate regulation as to quantity, absolute evenness for the whole length of the roller, 
regularity of flow, and for instant stoppage. The machines are in all cases driven by belts; in some roller-mills the secondary motions are created by belts, in others by geared wheels. In practical effect there does not appear to be any difference between. these modes.

Such are the general features of roller-mills, and there are several types in the market which, in a greater or less degree, satisfy all the conditions required. Every one of them has probably its weak point; and, provided that the material, 'size, and speed of the rollers. are alike, the pressure that can be applied to them without heating the bearings is the element that determines the amount of work that can be performed by the roller-mill; and that mill is best which, all other things being equal, will do the most duty in a given time.

It must not be supposed that the substitution of roller-mills for millstones is a simple matter of using one machine in place of another. On the contrary, no small amount of skill, in adjusting a roller-mill to the performance of the work, is required, and still greater skill in arranging a series of them to perform the work of gradual reduction. The roller-mill is, in point of fact, a tool that requires the intelligence of a skilful brain to use it efficiently. Whatever doubts there may be as to the advisability of the general adoption of the system of gradual reduction by rollermills, experience has shown that a roller-mill is an excellent machine for grinding semolina or middlings, especially if the purification of them is not quite perfect.

The most recent development of roller-milling is in the direction of substituting rollers for millstones in the low-grinding, or halfhigh-grinding system. A roller-mill has been brought out by Mr. J. A. A. Buchholz, consisting of three grooved rollers acting as two pairs, and under great pressure the wheat is reduced to flour and semolina in two operations. The wheat is fed between the first pair, which crush it severely; thence it passes to a sieve which takes out all the finer parts of the meal; what remains is carried to the second pair of rollers, which complete the grinding operation. The machines are in use in some large mills. For a dozen years or more combinations of as many as six or eight pairs of rollers with intermediate sifting machinery in one frame have been worked, and lately several roller-mills of this type have been. introduced. The complication of all the systems of grinding by roller-mills is what stands in the way of their general adoption, and the desideratum is, some form of mill which, while avoiding the defects of the millstones, will yet retain their simplicity of 
action : in fact, a machine that will at one operation obtain the same results as now require half a dozen.

It remains to speak of disintegrators. These, in various forms, have been tried for flour-making, but not with success. The violence of the operation, and the grinding or breaking of the bran which ensues, must inevitably spoil the quality of flour made by a disintegrator from any but tough-skinned wheats.

A combination of roller-mills and a modified disintegrator has been adopted by Nagel and Kaemp. The process consists in squeezing the wheat between smooth rollers running at equal speeds, thus fracturing the flour-bearing interior, which, when subjected to the dismembrator flies to pieces, the action of the dismembrator not being great enough to break up the bran. The process is repeated till the bran is cleaned. The semolina is reduced to flour in the ordinary way. Employed on some sorts of wheats the process is said to give good results.

One of the most important benefits arising from the use of rollers is undoubtedly the power they confer on the miller of removing, partly or entirely, at one stage or other of the grinding process, the germ of the wheat corn. Its presence in flour injures the quality, but it is an admirable food for cattle. If it escape all the preliminary stages, and appears in fragments or entire in the semolina, the smooth rollers flatten it out, and it can be easily removed by sifting. In millstone grinding it is cut up and mixed with the flour.

\section{Sifting or Dressing.}

The next machines in order of process are the dressing machines, which are used for sifting meal, semolinas, and flours. Up to about thirty years ago, the machine most generally employed was a cylinder formed of wire gauze, which was supported by wooden ribs, set in a slightly slanting position, inside which revolved, at a high speed, a shaft furnished with brushes which touched the wire. The meal was introduced at the upper end of the cylinder, and by the rotation of the brushes the floury particles were driven through the meshes of the gauze, while the offal, thoroughly freed from flour, escaped at the lower end.

About 1850, machines came into use in this country consisting of light reels of wooden laths, six to eight-sided, about 3 feet or 3 feet 6 inches in diameter, and from 12 feet to 35 feet in length, covered with silk gauze, through which the flour was sifted by causing the reel to rotate slowly so that the meal was lifted high 
enough to have a clear fall upon the silk on the lower side of the reel. This was a much gentler operation than the wire cylinder and brush, and had the advantage that the light fine particles of bran were not forced through the meshes, as they were with the brush machine; it had the disadvantage, however, of requiring much larger space than the old machines to do the same amount of work. To overcome this inconvenience there have been lately introduced centrifugal dressing machines. In these, cylinders of short length, covered with silk, are used, which are made to rotate; and the sifting is accomplished by throwing the meal lightly against the silk by means of beaters, which revolve at a somewhat rapid speed inside the cylinder of silk, with a certain space between the beaters and the silk. The rotation of the cylinder causes the meal to fall away from the silk as it reaches the upper side of the machine, to be again and again thrown against the silk, until, the fine particles having passed through the meshes of the gauze, the offal escapes at the tail. The advantage of the machines is the small space occupied in proportion to the work accomplished; while their drawbacks are, the wear and tear of silk, which is greater than in the reel machines. From their compact construction they are of much value, though in mills where there is ample available space they will not supersede the simple reel for dressing meal ground by millstones. But the use of the centrifugal machine is almost essential in dealing with meal ground from semolina by smooth-surface rollers; for although differential speed be adopted for the rollers, there is still a slight tendency to cake in the meal, especially in damp climates, and when tender wheats are used; and the beating of the centrifugal machine is in most cases sufficient to disintegrate the cakes, and thus to aroid the employment of a detacheur as a part of the roller-mill, or as a separate machine.

\section{Semolina or Middings Purifying.}

Passing from the dressing machinery to the next process, the most important machine of recent introduction is the purifier, so called because its purpose is to purify the semolinas, or middlings, from the small particles of bran which are mixed up with them, and to separate according to their density the lighter from the heavier semolinas. This work cannot be accomplished by any mere sifting operation, because where the gritty particles of semolina pass, then the bran particles, being of the same size, will pass also. But the bran particles, and the inferior quality of semolina being specifically 
lighter than the best and purest semolina, advantage is taken of this characteristic to divide them. The operation is assisted by the difference between the semolinas and bran in shape; the former are spheres or polyhedrons; the latter is always flat.

Until the invention and adoption of the purifiers the middlings, which are now recognised as the most valuable part of the grain for flour-making purposes, were commonly sold for making coarse biscuits, and even for cattle and pig feeding; simply because there was no known method of separating the branny particles from the good semolina or middlings. And in this country, the only system for the production of fine white flour was by the use of the highest qualities of white wheats ground and dressed very finely. These wheats then brought a considerably higher price in the market than red wheats, which in all other respects, except in the colour of the bread yielded by the flour made from them, were superior in quality; but the particles of the red or brown skin made the bread dark. With the use of the purifiers, better bread can be made from these red wheats than from the white wheats, because the discolouring particles of bran do not enter the flour, and consequently white wheats no longer fetch the high relative prices which they formerly commanded. But it must be borne in mind that it is only a power of more complete separation of the meal which these machines confer on the miller, and that if he be enabled by their use to manufacture a fine flour from the best of the materials which the machines separate for him, he still has to find a market for the inferior products of the machine. So that on the whole, though the advantages of the employment of purifiers are great in the manufacture of fine flour, yet, under the conditions on which in the United Kingdom the business of the miller is carried on, there are drawbacks which should make him cautious in adopting too extensively the use of this valuable machine. In all high-grinding and gradual-reduction processes its extensive use is indispensable.

The variety of purifiers in the market is very great, but they can all be classified under the following heads :-

(1.) Wind machines depending on the exposure of the material to currents of air.

(2.) Centrifugal machines where the separation is accomplished by centrifugal force, in combination with currents of air.

(3.) Sieve machines where the separation is accomplished by oscillating sieves in combination with currents of air.

The germ of the first-named class is the old Vienna wind 
machine, in which the semolina was exposed to a slight blast of wind, produced by a fan, and it was so contrived that the thin stream of impure semolina in its passage through the machine was two or three times subjected to the current of air which was sufficient to blow away some of the light particles into divisions prepared for them. But the semolina had to be repeatedly passed through the machine to make a good separation, and the work was never under complete control. Yet for some forty or fifty years this was the only purifier in use, and its employment was confined to the Austrian mills.

A great improvement was effected, still following the lines of this machine, by substituting exhaust for blast, and Charles Haggenmacher, of Budapest, contrived a series of machines of this type, which leave nothing to be desired. The stream of semolina is made to fall through a vertical rectangular trunk or pipe, fitted with baffling boards so fixed that in its descent the stream is caused to cross and recross repeatedly from one side of the pipe to the other, and a current of wind is drawn upwards through the pipe, so that as the semolina falls it is subjected to the action of the current again and again; the current is capable of being so regulated that it can be made to suck away either the whole stream of semolina, or only the lightest particles of bran, or any intermediate proportion; while, by an arrangement of airpassages and valves it is most ingeniously contrived that what has been sucked away is by a similar exposure to air-currents divided and redivided, so that the operator has the most complete control over the amount of purification of the semolina which he may desire to effect. The machines are very compact, and complete the work of purifying at one passage of the semolina through the machines. They are used universally in Austria and Hungary, but have not been extensively used in this country, because they are adapted only for the larger sizes of semolinas, and are not. fitted for the small sizes, or middlings, which British mills produce.

In the second class in which the purification is accomplished by centrifugal force in combination with currents of air, the machines vary only in details of construction, the principle being in all cases alike. The material to be separated is brought by a pipe to the centre of a horizontal revolving disk, by which it is thrown off in a circular shower depending on the speed of the disk and the specific weight of the semolina for its range. The heavier and better particles of semolina fly the farthest, the poorer and less heavy particles less far, and so in proportion, the quite light bran 
particles falling very near the disk. Receptacles with concentric divisions are constructed at various distances from the centre of the disk, into which the various qualities are thrown; but to assist the operation a current of air is established by an exhaust fan drawing downwards and towards the centre through the scattered semolina, which not only draws away with it the very lightest particles but also, by resisting the flight of the particles of medium weight, tends to deposit them in the inner divisions.

The third class of machine finds the greatest favour in the United Kingdom and America chiefly because it is best adapted to treat the smaller-sized semolinas. The principle of this type of machine is that it treats the semolina by running it over a long narrow sieve, which has a rapid oscillating or joggling motion; the semolina which falls on the sieve at the upper end is by this motion gently carried forward to the lower end of the sieve, and at the same time the result of the action is to cause all the light particles to find their way to the top of the stream, while the heavier particles sink to the surface of the sieve; the appearance of the stream in motion is as if slight ebullition was going on in it. The sieve is placed in a large chamber or case, and so connected with the sides as to form a horizontal diaphragm, dividing the case into two nearly equal parts ; it is covered with silk gauze with meshes graduated to the size of the semolinas under treatment, and, as just stated, the action of the sieve of itself tends to bring the particles of semolina down to the surface of the sieve through which they are intended to fall, and to float the light particles on the top of the stream. It remains to cause a current of air to pass upwards through the silk and through the layer of semolina on it, so balanced that it is just sufficient to keep any light particles from passing through the meshes of the sieve, while it is not so strong as to prevent the heavy particles from falling through. The current sweeps upwards through the chamber formed above the sieve to an outlet, and carries with it the very lightest and finest particles, and deposits them in a room constructed for the purpose, while the somewhat heavier of the branny particles are kept from passing through the meshes of the sieve, and float on the mass until they emerge over the tail into a receptacle prepared for them. The purified semolina that falls into the chamber below the sieve is collected and carried out of the machine by a worm. The management of the air-current is the great point of importance, and has taxed to the utmost the ingenuity of inventors. The current is produced either by a blast fan in connection with the chamber under the sieve, or by 
exhausting the chamber above the sieve, or by both in combination, by making appropriate inlets and outlets in each case. The great difficulty has been to obtain an even pressure of air over the whole area of the underside of the sieve, graduated and capable of being regulated according to the nature of the semolina and to its quantity, and capable of variation at different parts of the sieve; and a pressure which, while sufficient for its purpose, is not so great as to carry away at the outlet of the air-current, and thus waste, any large quantity of material. A second point is the difficulty of keeping the meshes of the sieve open, as they are prone to be stopped up by particles just large enough to enter the meshes and not large enough to pass through. Gentle brushing by travelling brushes has been adopted to overcome this difficulty, by which the sieve is kept open. The practical working of the purifiers has also developed innumerable minor impediments, which by one inventor or another have been conquered, and the best machines now in the market are capable of successfully purifying the finest and softest middlings, even if they approach in character to flour, without material waste. This type of machine has its origin in a French machine, constructed by Cabane. But the Americans have greatly improved on the original, and have taken out numerous patents for details and improvements of purifiers during the last few years. It is obvious that in all purifiers, as the separation is made by gentle air-currents, the particles to be separated must not differ much in size. It is therefore necessary to provide a series of purifiers in mills, to each of which should be allotted a separate size of semolina. The semolinas are sized by sifting through silk gauze. An even foed to the machines is also of great importance.

The use of purifiers is comparatively small in this country, because the conditions of the trade are not adapted generally for the manufacture of the finest flour. In Hungary and in America, however, there are to be found regular systems of machines whereby the semolina is purified and repurified, and whereby what is rejected from the first purification is again and again treated. This involves the employment of a very large number of purifiers, a number indeed so great that it is impossible to find space for them in a mill, unless it has been specially built for the purpose. One of the chief obstacles with millers to the adoption of any system of which purification and repeated grindings and dressings form a part is very often that the buildings at their command do not afford the necessary space. But if fine flours are to be made, abundance of purifying power is 
an absolute necessity, and the purifier may claim to be a more important machine than the roller-mill in any modern process; for the work of the latter may be performed by the millstone, while without the purifier neither millstone nor roller-mill can make the finest flours.

Such are the features of the principal machines in use $j$ in modern corn-mills; and the property that is common to all of them is that no one machine of the series is confined to the performance of one exact and particular duty; but in every case, and at every stage of the manufacture of flour, there is abundant freedom, and indeed actual need, for the display of skill on the part of the operator in the control and regulation of the duty thrown on each machine. Thus, in the grinding operations, whether by millstones or by rollers, the utmost skill is required to adapt the treatment both to the quality and character of wheat in use, and to the ultimate result in flour which it is desired to attain. And upon a very little difference in this operation may depend the profit or loss of the miller. So in the matter of dressing the meal, the variations of practice are most numerous, some beginning where others leave off, and vice versâ. Again, in handling purifiers, apparently the most opposite opinions prevail as to the best mode of procedure. The mere possession of one machine, or any series of machines, does not constitute a process or a system, as each and all may be handled in a great variety of ways, and the same machines can be made to produce the most varied results. The handling of the machines described is, therefore, purely a practical and technical matter upon which no hard and fast rules can be laid down.

Again, the arrangement and position of the machines in the mill building affords scope for much ingenuity. In this matter, however, one rule must predominate over all other considerations, viz., that the material in process of manufacture must not require to be moved by hand-labour at any stage from its reception into the mill until it is finally packed in the bags in which it is to be delivered to the consumer. Appliances in the way of endless belting, worms, conveyers, pneumatic apparatus, \&c., are available for carrying in horizontal directions, while the elevator, consisting of cups fixed on an endless band, serves for lifting in a vertical direction.

Wherever practicable it is always sought to so arrange that the stuff may run by gravity from one machine to another, and it is for this reason that the buildings for flour-mills are generally lofty. 
Sifting operations perform a great part in the manufacture, and the distinctions in size that it is necessary to make are often of a minute kind. The adoption of silk gauze as the material for sifting has alone rendered these fine distinctions possible; and a sketch of modern milling would be incomplete which did not give the manufacturers of these silk cloths the highest credit for the beauty and regularity of their fabrics. When it is considered that they are called upon to supply silk cloth containing certain definite and known numbers of holes to the square inch, varying from two hundred and fifty up to about twenty-three thousand, and that in the higher numbers the holes are quite microscopical though regular in size, while at the same time the gauze has to be of sufficient strength to bear the strain that is put upon it, the difficulty of their task can be conceived. The best quality of silk gauzes is manufactured in Switzerland.

Finally, in spite of agitation in the direction of persuading people to eat brown bread or bread containing the skin of the grain, the tendency, though not moving rapidly, is clear and marked on the observation of a series of years towards the consumption of finer and whiter bread by the masses. Popular taste fluctuates, but should it continue to move in the same direction a steady spread of the use of high-grinding systems may be looked for. 